\title{
Delayed Appearance of Conduction Block in Multifocal Motor Neuropathy-A Case Report
}

\author{
Leila Darki and Said R Beydoun \\ Neuromuscular Division, Keck School of Medicine, University of Southern California, Los Angeles, CA, US
}

\begin{abstract}
ntroduction: Multifocal motor neuropathy (MMN) is a rare, treatable, immune-mediated neuropathy often associated with multifocal conduction block (CB). The hallmark electrodiagnostic feature is the presence of CB occurring at non-entrapment sites. However, MMN without CB has also been described and can be diagnosed, even in the absence of CB. Therefore, it is crucial to diagnose and identify MMN cases without $\mathrm{CB}$, as it is a treatable disorder. Case presentation: We present a case with progressive symptoms of asymmetric distal upper and lower extremity weakness with no sensory deficits. Intravenous immunoglobulin (IVIG) therapy was initiated, as the patient fulfilled the criteria for probable MMN, despite the absence of CB. The patient's symptoms demonstrated a relative plateau phase in response to IVIG. Although the patient lost follow-up visits, repeated electrodiagnostic study, conducted 11 years after initial presentation, revealed new CB in nerve segments that previously did not show any evidence of $\mathrm{CB}$. Conclusion: This case emphasizes the importance of early diagnosis and respectively initiating early IVIG treatment in MMN, in order to maintain the clinical function. Underdiagnosis of clinically suspected MMN, based on absence of CB, will result in denial of treatment to potential IVIG responders.
\end{abstract}

\section{Keywords}

Multifocal motor neuropathy, conduction

block, intravenous immunoglobulin

Disclosure: Said R Beydoun has received grant research support from Alexion, Argenx, Daiichi Pharma, and Pfizer. He has served as an advisory board member for Grifols, MT Pharma and Sarepta, and is a speaker for Grifols. Leila Darki has nothing to disclose in relation to this article.

Compliance with Ethics: All procedures were followed in accordance with the responsible committee on human experimentation and with the Helsinki Declaration of 1975 and subsequent revisions. Written informed consent was not obtained from the patient for publication of this case report due to loss of follow-up; no identifying information or images were used in the publication of this paper.

open Access: This article is published under the Creative Commons Attribution Noncommercial License, which permits any non-commercial use, distribution, adaptation and reproduction provided the original author(s) and source are given appropriate credit.

Received: June 12, 2017

Accepted: August 2, 2017

Citation: US Neurology, 2017;13(2):99-102

Corresponding Author: Leila Darki, Neuromuscular Division, Keck School of Medicine, University of

Southern California, 1520 San Pablo St, Suite 3000, Los

Angeles, CA 90033, US. E: Leila.darki@med.usc.edu
Multifocal motor neuropathy (MMN) is a rare, pure, motor neuropathy characterized by slowly progressive, asymmetric, predominantly distal weakness, commonly of the upper limbs, and may cause considerable disability. ${ }^{1,2}$ Presence of multifocal motor conduction block (CB) at locations other than common entrapment sites, with normal sensory nerve conduction in the corresponding segments, is the hallmark of MMN, but some patients with typical MMN have no detectable CB. ${ }^{2-4}$

Although, the pathophysiology underlying CB and other nerve dysfunction in MMN is not exactly clear, the node of Ranvier and its surrounding structures likely play an important role in $\mathrm{MMN}^{.5-7}$ In addition, clinical response to immune modulation therapy and elevated titers of anti-ganglioside antibodies, such as immunoglobulin M (IgM) anti-GM1 antibodies in 30-88\% of patients with MMN, support an immune-mediated etiology. ${ }^{8-10}$

The absence of $\mathrm{CB}$ in some patients may represent limitations of electrodiagnostic testing for identification of $C B$ at very proximal or distal nerve segments. ${ }^{3,4}$ It may also represent activity-dependent $\mathrm{CB}(\mathrm{ADCB})$, which is not detectable by routine electrodiagnostic studies. The pathophysiology of ADCB has been attributed to $\mathrm{Na}^{+}-\mathrm{K}^{+}$pump-induced axonal hyperpolarization. ${ }^{11}$

MMN is a treatable disorder, and intravenous immunoglobulin (IVIG) is currently considered the standard treatment. ${ }^{12-15}$ The goal of treatment is to reverse the motor CB and limit axonal injury which can lead to irreversible functional impairment. ${ }^{16}$ This underscores the need for an early diagnosis and treatment. ${ }^{1}$ In the setting of an MMN phenotype presentation, it is extremely vital to identify those patients who don't have CB as those would show similar response to IVIG treatment. ${ }^{17}$ Moreover, it has been shown that better long-term outcome depends on early diagnosis and treatment. ${ }^{1,18,19}$

\section{Case presentation}

The patient was a 32-year-old, Hispanic female, who initially presented with progressive loss of hand dexterity, which resulted in limitations of activities of daily living (ADL). Her symptoms manifested initially as right-hand weakness, described as inability to hold coins as well as difficulty with typing and writing. Symptoms progressed to involve the left upper extremity within a few months. 
Table 1: Nerve conduction studies

\begin{tabular}{|c|c|c|c|c|c|c|c|}
\hline \multicolumn{4}{|l|}{2005} & \multicolumn{4}{|l|}{2016} \\
\hline Nerve & Latency (ms) & Amplitude (mv) & $\mathrm{CV}(\mathrm{ms})$ & Latency (ms) & Amplitude (mv) & $\mathrm{CV}(\mathrm{ms})$ & Area (\%) \\
\hline Median R & $\mathrm{N}<4.4$ & $N>5.0$ & $N>49.0$ & $\mathrm{~N}<4.4$ & $N>5.0$ & $N>49.0$ & \\
\hline Wrist & 4.2 & 12.9 & & 3.9 & 4.6 & & 100.0 \\
\hline Elbow & 7.3 & 12.8 & 59.6 & 10.9 & 1.9 & 28.0 & 43.7 \\
\hline Upper arm & $\mathrm{N} / \mathrm{A}$ & $\mathrm{N} / \mathrm{A}$ & N/A & 12.7 & 1.8 & 41.0 & 38.4 \\
\hline Ulnar R & $N<4.4$ & $N>5.0$ & $N>49.0$ & $N<4.4$ & $N>5.0$ & $N>49.0$ & \\
\hline Wrist & 2.4 & 12.1 & & 2.4 & 8.3 & & 100.0 \\
\hline Below elbow & 4.9 & 12.1 & 56.0 & 5.9 & 7.2 & 54.0 & 89.1 \\
\hline Above elbow & 6.7 & 12.0 & 61.1 & 7.9 & 6.2 & 49.0 & 77.6 \\
\hline Tibial R & $N<5.8$ & $N>4.0$ & $N>40.0$ & $\mathrm{~N}<5.8$ & $N>4.0$ & $N>40.0$ & \\
\hline Ankle & 5.0 & 12.7 & & 4.6 & 9.7 & & 100.0 \\
\hline Pop fossa & 13.2 & 10.8 & 45.1 & 11.4 & 1.5 & 47.0 & 23.0 \\
\hline Peroneal R & $\mathrm{N}<6.4$ & $N>2.2$ & $N>40.0$ & $\mathrm{~N}<6.4$ & $\mathrm{~N}>2.2$ & $N>40.0$ & \\
\hline Ankle & 4.7 & 7.7 & & 4.4 & 7.3 & & 100.0 \\
\hline Fibula & 9.8 & 7.1 & 50.9 & 10.3 & 4.7 & 47.0 & 75.4 \\
\hline Pop fossa & N/A & N/A & N/A & 11.5 & 4.2 & 71.0 & 72.0 \\
\hline
\end{tabular}

$C V=$ conduction velocity; $m s=$ millisecond $; m v=$ millivolt; $N=$ normal; $N / A=$ not assessed $;$ Pop fossa = popliteal fossa; $R=$ right .

Her weakness continued to progress to the point that she had right wrist drop within 3 months. She reported gait difficulty with slight right foot drop. She denied pain or sensory complaints. Neuromuscular examination revealed decrease bulk in the forearm extensors, bilaterally. Manual muscle-strength testing revealed moderate asymmetric weakness in the wrist extensors, differential weakness in the finger extensors, and milder weakness in the wrist flexors, finger flexors, intrinsic hand muscles, and right ankle dorsiflexors. Deep tendon reflexes were absent in the upper extremities. Sensory examination was normal. Her overall disability sum score (ODSS) was $5(3+2)$.

Nerve conduction studies (NCS) showed normal compound muscle action potential (CMAP) distal amplitudes with no evidence of $\mathrm{CB}$ in all tested nerves (Table 1). The proximal upper extremities segments were not checked. Sensory potentials were preserved. F wave was absent in bilateral, median, and right peroneal nerves. On electromyographic examination, slight chronic denervation changes, with decreased number of functioning motor unit potentials, were noted in the multiple muscles including the right extensor digitorum communis, right abductor pollicis brevis, right biceps brachii, left extensor digitorum communis, left biceps brachii, and the left abductor pollicis brevis. Serum GM1 antibodies were negative. Serum creatine kinase was mildly elevated.

Based on her clinical presentation, weakness topography, and distribution, probable MMN was diagnosed, despite the absence of CB and negative serology. Following a diagnosis, the patient was treated with IVIG, at a dose of $1 \mathrm{~g} / \mathrm{kg}$ monthly. She did show a positive response, with improvement in her ability to do certain upper extremity ADLs, and partial improvement in her strength. Her ODSS score improved to $3(2+1)$.

Patient lost to follow-up for many years, but continued maintenance IVIG treatment, under supervision of her primary neurologist. On reevaluation, 11 years after initial presentation, the patient indicated that her motor symptoms have partially improved with IVIG, and has been able to maintain the improvement. She also experienced treatment benefit wear-off toward the end of the treatment cycle. Neurologic examination revealed slightly decreased bulk in the right first web space and lateral thenar area. Manual muscle strength testing showed moderate weakness in the intrinsic hand muscles and wrist extensors, and mild asymmetric weakness in the finger extensors and ankle dorsiflexors. Hand-held dynamometry strength was $11.1 \mathrm{~kg}$ in the right hand, and $19.5 \mathrm{~kg}$ in the left hand. Gait was normal except for slight difficulty with walking on right heel. Upper extremity deep tendon reflexes were decreased but present. Her ODSS score was $3(2+1)$.

Repeat NCS revealed new CB in previously normal segments of right median and posterior tibial nerves, and probable $\mathrm{CB}$ in right peroneal nerve (Table 1, Figure 1). There was also evidence of axonal loss in the right median and to a lesser degree in the right ulnar and the right tibial nerves, manifesting as relatively low distal CMAP amplitude, in comparison to the initial study. Moderate amount of fibrillation potentials, and positive sharp waves, were noted in the right extensor digitorum communis, first dorsal interosseous, and abductor pollicis brevis. Chronic denervation changes, and decreased number of functioning motor unit potentials, were seen in multiple sampled muscles in the upper extremities and distal right lower extremity.

\section{Discussion}

MMN, a treatable motor neuropathy presents with slowly or stepwise progressive, asymmetric weakness in the more than one motor nerve distribution without objective sensory loss and absence of upper motor neuron signs. The diagnosis of MMN is based on clinical, laboratory and electrophysiological characteristics. The European Federation of Neurological Societies/Peripheral Nerve Society has established clinical and electrophysiological criteria for diagnosis of MMN. Based on these guidelines, definite $\mathrm{CB}$ in one motor nerve is required to diagnose definite 
MMN. Definite CB is defined as negative peak CMAP area reduction on proximal versus distal stimulation of at least $50 \%{ }^{20}$

Our patient satisfied the core clinical criteria for MMN (weakness in the distribution of more than two nerves); however, the initial electrodiagnostic study revealed normal distal CMAP amplitudes with no evidence of CB. As the patient's history and physical examination were suggestive of MMN, treatment with IVIG was started despite the absence of the typical electrodiagnostic features. Clinical objective improvement with IVIG further validated the diagnosis of MMN. Finally, the appearance of new $C B$ in previously normal segments, years after the initial presentation confirmed the diagnosis of definite MMN. ${ }^{20}$ Although there is a possibility that initial study missed the $\mathrm{CB}$, as proximal upper extremities were not assessed, the interesting point of this case is the appearance of $C B$ in same segments that previously did not show any evidence of $\mathrm{CB}$.

Our patient had improvement in both muscle strength and ODSS which was mainly due to early recognition and initiation of appropriate treatment.

Van den Berg-Vos et al. studied 38 patients with MMN who had never received IVIG treatment, with a disease duration ranging from 6 months to 34 years. They showed that patients with a long disease duration had significantly more severe weakness, disability and electrophysiological abnormalities than patients with a short disease duration. ${ }^{18}$

Although the pathology underlying MMN is not well described, the evidence suggests that demyelination, remyelination, axonal regeneration, collateral sprouting, and axonal loss do all occur in patients with $\mathrm{MMN}^{2}{ }^{2}$ Progression of MMN and its underlying mechanisms can be evaluated by means of follow-up electrodiagnostic study. ${ }^{21}$ To our knowledge, long-term electrophysiological follow-up studies of patients with MMN are limited. In our case, the electrodiagnostic study revealed significant changes after a long period of lost follow-up. New definite CB (CMAP area reduction on proximal versus distal stimulation of at least $50 \%$ ) was detected in right median and tibial nerves. This represented an area of newly appearing $\mathrm{CB}$ in previously normal segments. Although, the inherent technical difficulty of stimulation at the popliteal fossa may account for some degree of amplitude drop and dispersion in tibial nerve, corresponding weakness in tibial innervated muscles and absence of CB in the initial study, favor new true $\mathrm{CB}$. There was reduction in the distal CMAP amplitudes in the right median nerve and to a lesser degree in the right ulnar and tibial nerves, when compared with the prior electrodiagnostic study. This likely indicates ongoing axonal degeneration even as the patient was receiving maintenance IVIG therapy. This is also supported by the presence of active denervation and reinnervation changes on electromyographic examination.

These findings are also consistent with what has been reported by Van den Berg-Vos, describing the occurrence of axonal loss in MMN over the years despite IVIG treatment.19 In this study, they followed up 11 patients with MMN, who received maintenance IVIG every 1-7 weeks, on average dose
Figure 1: Conduction block in the tibial, peroneal, and median nerves

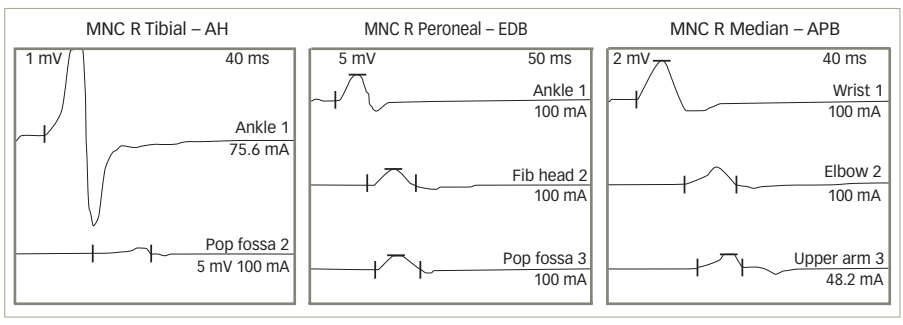

$A H=$ abductor hallucis; $A P B=$ abductor pollicis brevis; $E D B=$ extensor digitorum brevis; Fib head = fibular head; $M N C=$ motor nerve conduction; Pop fossa = popliteal fossa; $R=$ right.

of 7-48 g per week, for 4-8 years. Muscle strength improved significantly within 3 weeks of the start of IVIG treatment, and was still significantly better at the last follow-up than before treatment, but it decreased slightly yet significantly during the follow-up period. The electrodiagnostic study was performed before treatment and each year during IVIG maintenance treatment. They suggested that IVIG "favorably influences the mechanism of demyelination and remyelination or reinnervation, but axonal loss cannot be prevented."19 However, Vucic indicated that higher doses of maintenance IVIG treatment for many years can prevent both clinical and electrodiagnostic worsening. ${ }^{22}$

\section{Conclusion}

MMN is a rare, treatable neuropathy, but good long-term outcome is dependent on early treatment. It is therefore important to diagnose MMN and differentiate it from other conditions. The clinical presentation of MMN can mimic several neurological conditions, including those with poor prognoses, such as motor neuron disease. Serological evidence of anti-GM1 antibodies and electrodiagnostic findings of CB are helpful diagnostic clues for MMN, but not always present. Importantly, MMN patients without CB can demonstrate similar robust response to IVIG, hence early diagnosis and initiation of IVIG treatment is crucial.

In summary, our case illustrates the following important points, known about MMN:

- the importance of diagnosing MMN even without $\mathrm{CB}$, when the clinical history and pattern of motor findings suggest the diagnosis and initiation of early immunoglobulin therapy;

- it also indicates that CB can appear many years later, despite patients showing positive response to the treatment;

- it illustrates the slow, progressive nature of the disease, as evident by progressive axonal loss; and

- more importantly, had treatment been denied because of absence of CB on the initial study, her course would have been significantly different with development of fixed irreversible axonal loss and resultant muscle weakness and atrophy. $\mathrm{u}$
1. Cats EA, van der Pol WL, Piepers S, et al., Correlates of outcome and response to IVIg in 88 patients with multifocal motor neuropathy, Neurology, 2010;75:818-25.

2. Hahn AF, Beydoun SR, Lawson V, et al., A controlled trial of intravenous immunoglobulin in multifocal motor neuropathy, J Peripher Nerv Syst, 2013;18:321-30.

3. Katz JS, Barohn RJ, Kojan S, et al., Axonal multifocal motor neuropathy without conduction block or other features of demyelination, Neurology, 2002;58:615-20.

4. Pakiam AS, Parry GJ, Multifocal motor neuropathy without overt conduction block, Muscle Nerve, 1998;21:243-5.

5. Lawson VH, Arnold WD, Multifocal motor neuropathy: a review of pathogenesis, diagnosis, and treatment, Neuropsychiatr Dis Treat 2014;10:567-76.

6. Susuki K, Rasband MN, Tohyama K, et al., Anti-GM1 antibodies cause complement-mediated disruption of sodium channel clusters in peripheral motor nerve fibers, $J$ Neurosci, 2007;27:3956-67.

7. Kaji R, Physiology of conduction block in multifocal motor neuropathy and other demyelinating neuropathies, Muscle Nerve, 2003;27:285-96.

8. Vlam L, van der Pol WL, Cats EA, et al., Multifocal motor neuropathy: diagnosis, pathogenesis and treatment strategies, Nat Rev Neurol, 2011;8:48-58. 
9. Vlam L, van den Berg LH, Cats EA, et al., Immune pathogenesis and treatment of multifocal motor neuropathy, I Clin Immunol, 2013;33 Suppl 1:S38-42.

10. Rison R, Beydoun SR, Differentiating multifocal motor neuropathy from entrapment neuropathy — a diagnostic challenge, US Neurology, 2016;12:17-21.

11. Kaji R, Bostock H, Kohara N, et al., Activity-dependent conduction block in multifocal motor neuropathy, Brain, 2000;123:1602-11.

12. Azulay JP, Blin O, Pouget J, et al., Intravenous immunoglobulin treatment in patients with motor neuron syndromes associated with anti-GM1 antibodies: a double-blind, placebo-controlled study, Neurology, 1994;44:429-32.

13. Van den Berg LH, Kerkhoff $\mathrm{H}$, Oey PL, et al., Treatment of multifocal motor neuropathy with high dose intravenous immunoglobulins: a double blind, placebo controlled study, J Neurol Neurosurg Psychiatry, 1995;59:248-52.

14. Federico P, Zochodne DW, Hahn AF, et al., Multifocal motor neuropathy improved by IVIg: randomized, double-blind, placebo-controlled study, Neurology, 2000;55:1256-62.

15. Léger JM, Chassande B, Musset L, et al., Intravenous immunoglobulin therapy in multifocal motor neuropathy: a double-blind, placebo-controlled study, Brain, 2001;124:145-53.

16. Van Asseldonk JT, van den Berg LH, Kalmijn S, et al., Axon loss is an important determinant of weakness in multifocal moto neuropathy, I Neurol Neurosurg Psychiatry, 2006;77:743-7.

17. Delmont E, Azulay JP, Giorgi R, et al., Multifocal motor neuropathy with and without conduction block: a single entity?, Neurology, 2006;67:592-6.

18. Van den Berg-Vos RM, Franssen $\mathrm{H}$, Visser J, et al., Disease severity in multifocal motor neuropathy and its association with the response to immunoglobulin treatment, J Neurol, 2002;249:330-6.
19. Van den Berg-Vos RM, Franssen $\mathrm{H}$, Wokke $\mathrm{JH}$, van den Berg LH, Multifocal motor neuropathy: Iong-term clinical and electrophysiological assessment of intravenous immunoglobulin maintenance treatment, Brain, 2002;125:1875-86.

20. Joint Task Force of the EFNS and the PNS, European Federation of Neurological Societies/Peripheral Nerve Society Guideline on management of multifocal motor neuropathy: report of a joint task force of the European Federation of Neurological Societie and the Peripheral Nerve Society--first revision, J Peripher Nerv Syst, 2010;15:295-301.

21. Taylor BV, Wright RA, Harper CM, Dyck PJ, Natural history of 46 patients with multifocal motor neuropathy with conduction block, Muscle Nerve, 2000;23:900-8.

22. Vucic S, Black KR, Chong PS, Cros D, Multifocal motor neuropathy: decrease in conduction blocks and reinnervation with long-term IVIg, Neurology, 2004;63:1264-9. 